\title{
EFEITO DA ESTIMULAÇÃO ELÉTRICA NEUROMUSCULAR (EENM) NO MÚSCULO AGONISTA E ANTAGONISTA DE INDIVÍDUOS COM HEMIPLEGIA ESPÁSTICA DECORRENTE DE DISFUNÇÃO VASCULAR ENCEFÁLICA: REVISÃO SISTEMÁTICA
}

\author{
EFFECT OF NEUROMUSCULAR ELECTRICAL STIMULATION (NMES) ON AGONIST \\ AND ANTAGONIST MUSCLES OF INDIVIDUALS WITH SPASTIC HEMIPLEGIA \\ RESULTING FROM CEREBRAL VASCULAR DYSFUNCTION: SYSTEMATIC REVIEW
}

\author{
Djenifer Queiroz de Souza ${ }^{1}$ \\ Izabela dos Santos Mendes ${ }^{1}$ \\ Ana Carolina Lacerda Borges ${ }^{1}$ \\ Sérgio Takeshi Tatsukawa de Freitas ${ }^{2}$ \\ Fernanda Pupio Silva Lima ${ }^{3}$ \\ Mário Oliveira Lima ${ }^{3}$ \\ Paulo Roberto Garcia Lucareli ${ }^{4}$
}

RESUMO: O quadro clínico predominante nos pacientes com sequelas de lesão de motoneurônio superior após Acidente Vascular Encefálico (AVE) é a hemiplegia espástica, levando a limitações funcionais e incapacidades contralaterais ao hemisfério lesado. As pesquisas em neurorreabilitação vêm avançando, contribuindo, assim, com os parâmetros ideais a serem utilizados nos recursos terapêuticos, visando à diminuição da hipertonia espástica e o aumento da força muscular dos pacientes hemiplégicos. Entre estes recursos destaca-se a Estimulação Elétrica Neuromuscular (EENM), a qual provê o controle da hipertonia, relaxamento do músculo espástico e estimulação sensorial de vias aferentes modificando a propriedade elástica dos músculos e recrutando fibras musculares, podendo melhorar sua funcionalidade. O objetivo deste estudo foi realizar uma revisão sistemática acerca dos efeitos da estimulação elétrica neuromuscular (EENM) quando aplicada no músculo agonista e antagonista de indivíduos hemiplégicos espásticos pós-disfunção vascular encefálica (DVE). Para tal realizou-se um levantamento bibliográfico de janeiro a outubro de 2011, nos bancos de dados da SciELO, Bireme, Lilacs e PubMed, bem como, na literatura disponível no acervo da biblioteca central da Universidade do Vale do Paraíba, sendo analisados artigos no período de 1993 a 2010. Os artigos analisados sugerem que tanto a EENM antagonista quanto a agonista são um método de tratamento útil na reabilitação de indivíduos hemiplégicos espásticos, porém há escassez literária quanto a ensaios clínicos comparativos de tais métodos, sendo este um campo de estudo importante para a efetividade da intervenção fisioterapêutica visando à reabilitação dos pacientes hemiplégicos.

Palavras-chave: eletroestimulação; acidente vascular encefálico; espasticidade.

ABSTRACT: The predominant clinical picture in patients with upper motoneuron injury after a Stroke is spastic hemiplegic, leading to functional limitations and disabilities contralateral to the damaged hemisphere. Research on neurorehabilitation has advanced the optimal parameters used in therapeutic resources, aiming to decrease

\footnotetext{
${ }^{1}$ Graduanda em Fisioterapia - UNIVAP.

${ }^{2}$ Docente da UNIVAP. Mestre em Ciências Biológicas, doutorando em Engenharia Biomédica.

${ }^{3}$ Doutor em Engenharia Biomédica e docente da UNIVAP.

${ }^{4}$ Docente da Universidade Nove de Julho - SP. Doutor em Ciências (Fisiopatologia Experimental) - Universidade de São Paulo-USP. Fisioterapeuta senior do Hospital Israelita Albert Einstein - Laboratório de Estudos do Movimento Humano Einstein - LEME.

E-mail: paulolucareli@uninove.br
} 
um padrão flexor dos membros superiores (rotação interna e adução do ombro, flexão do cotovelo, pronação e flexão do punho e dedos) e extensor dos membros inferiores (extensão e rotação interna do quadril, extensão do joelho, flexão plantar e inversão do pé) (LIANZA, 2001; TEIVE, 1998).

Os pacientes com sequelas de DVE apresentam ainda, redução do número de unidades motoras recrutadas, resultando em contração muscular ineficiente e limitação do desempenho funcional (GLANZ et al., 1996).

Assim, programas de fortalecimento muscular, se fazem necessários na reabilitação de tais indivíduos, pois auxiliam no restabelecimento das funções normais do músculo e na redução da espasticidade (NORONHA, 1997).

A análise quantitativa das condições anteriormente descritas pode ser obtida por meio da eletromiografia (EMG), usada para mensurar a atividade elétrica do músculo e, consequentemente, avaliar a espasticidade. A EMG é um método para detectar, medir e registrar os potenciais de ação de fibras musculares produzidos durante uma contração, permitindo interpretações em condições normais e patológicas sendo utilizada como indicador de estresse, parâmetro de controle do sistema nervoso e reconhecimento de padrões de movimento (SORINOLA, 2009; MAURITZ, 2004).

Outro instrumento fundamental na avaliação do desempenho muscular e da espasticidade é o dinamômetro isocinético, considerado um equipamento eficaz, pois favorece a padronização da angulação, velocidade de estiramento e posicionamento, minimizando a subjetividade da avaliação (SORINOLA, 2009; MINUTOLI et al., 2007; FREITAS, 2005).

Atualmente, observa-se o avanço das pesquisas em neuroreabilitação, contribuindo com os parâmetros ideais a serem utilizados nos recursos terapêuticos. Vários destes recursos são empregados com o objetivo de diminuir a hipertonia espástica e aumentar a força muscular, entre os quais destaca-se a Estimulação Elétrica Neuromuscular (EENM).

A EENM é empregada para a contração de músculos plégicos ou paréticos com objetivos de ganho de força e funcionalidade, podendo ser utilizada também para controlar a hipertonia espástica, devido ao mecanismo de inibição recíproca, relaxamento do músculo espástico e estimulação sensorial de vias aferentes, por meio da neuroplasticidade, modificando, assim, a propriedade elástica dos músculos e recrutando fibras musculares (GLINSK; HARVEY, 2007; LIMA et al.; MIYAZAKI, 2008).

Habitualmente utiliza-se, como protocolo, a aplicação da EENM no músculo antagonista ao espástico.

Schuster et al. (2007) aplicaram a Estimulação Elétrica Funcional (FES) no músculo tibial anterior parético com frequência de $50 \mathrm{~Hz}$ e largura de pulso de 250us, quantificando a força muscular, o tônus, a amplitude de movimento, parâmetros espaços-temporais da marcha e pressão plantar, observando melhora da amplitude de movimento (ADM) ativa e passiva da dorsiflexão, da reeducação muscular, diminuição da espasticidade e 
melhor distribuição de peso do lado afetado com consequente melhora da marcha.

Deste modo, Jerônimo et al. (2007), ao analisarem a marcha de 5 crianças com quadro clínico de hemiparesia, após aplicação de EENM no músculo tibial anterior, verificaram que todas as crianças apresentaram diferenças menores entre 0 comprimento do passo plégico e não plégico.

Nunes et al. (2008) também aplicaram a EENM no músculo tibial anterior parético espástico de 10 pacientes, evidenciando melhora da força muscular e da ADM, sugerindo que a EENM pode ser útil no auxilio terapêutico.

Entretanto, alguns estudos se dispuseram a analisar a aplicação da EENM no músculo espástico.

Lima et al. (2008) aplicaram a EENM no músculo espástico extensor do joelho dos indivíduos hemiparéticos, resultando em diminuição da espasticidade e aumento de força dos extensores do joelho do lado parético.

Silva et al. (2010) analisaram a fadiga e a força muscular, por meio do teste de força muscular e eletromiografia do músculo espástico (bíceps braquial), após a EENM em cima de 2 protocolos, com frequência de $50 \mathrm{~Hz}$ e $2000 \mathrm{~Hz}$, respectivamente. Os resultados não evidenciaram o aparecimento de fadiga muscular, porém constatou-se melhora da força muscular.

Portanto, a aplicação da estimulação elétrica funcional pode desenvolver uma melhora de longo prazo, contribuindo com a reabilitação física desses pacientes, todavia com métodos de aplicação ainda controversos.

\section{OBJETIVO}

O objetivo deste estudo foi realizar uma revisão sistemática sobre os efeitos da estimulação elétrica neuromuscular (EENM) quando aplicada no músculo agonista e antagonista de indivíduos hemiplégicos espásticos pós-disfunção vascular encefálica (DVE).

\section{METODOLOGIA}

Foi realizada uma revisão sistemática e descritiva no banco de dados LILACS, SciELO, PubMed, MEDLINE e BIREME, bem como na literatura disponível no acervo da biblioteca central da Universidade do Vale do Paraíba (UNIVAP).

Utilizou-se as seguintes palavras chave: Acidente Vascular Encefálico, Espasticidade, Estimulação Elétrica Funcional.

A literatura pesquisada totalizou 534 obras, as quais foram publicadas em português, inglês e espanhol, no período de 1993 a 2011.

Os critérios de inclusão envolveram os trabalhos relacionados à EENM, DVE, hipertonia espástica, inibição recíproca, inibição autógena, eletromiografia e dinamometria isocinética. A partir do qual obtiveram-se 43 obras, devidamente citadas na referência.

Foram excluídas as literaturas publicadas antes de 1990 e aquelas cuja metodologia não ficou bem esclarecida. 


\section{RESULTADOS}

Os trabalhos utilizados foram agrupados de acordo com o assunto tratado, conforme demonstrado na Fig. 1, cujos valores excedem $100 \%$, pois algumas literaturas compartilham assuntos.

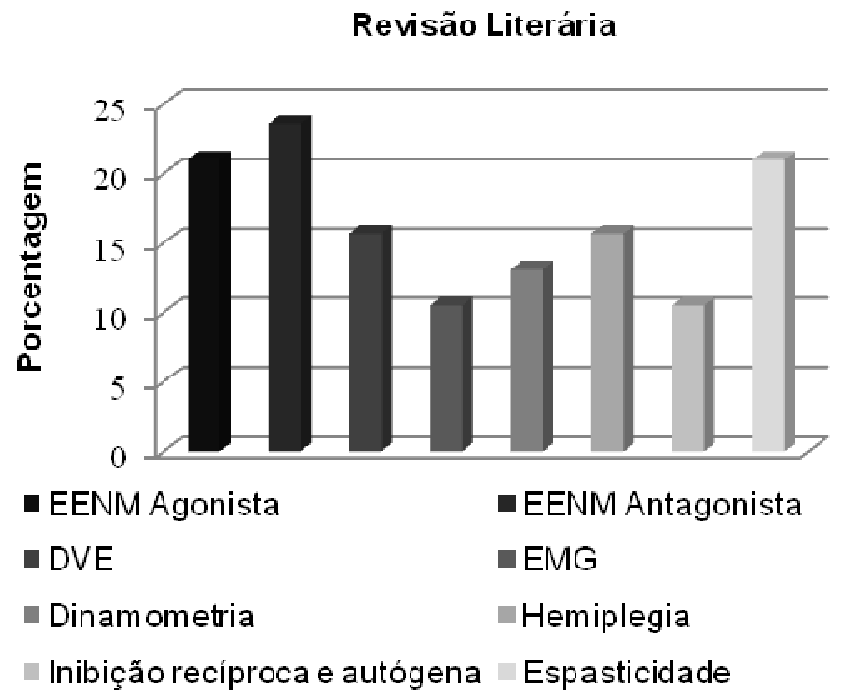

Fig. 1 - Distribuição porcentual da literatura pesquisada, conforme assunto.

Entre os artigos pesquisados alguns descreviam 0 uso da EENM sobre a musculatura espástica (agonista) e outros relatavam sua aplicação sobre a musculatura antagonista, conforme indicado, respectivamente, nas Tabelas 1 e 2 .

Tabela 1 - Distribuição dos artigos relacionados à EENM agonista

\begin{tabular}{|c|c|c|c|}
\hline AUTOR & ANO & $\begin{array}{l}\text { OBJETIVO } \\
\end{array}$ & RESULTADOS \\
\hline Newsam; Baker & 2004 & $\begin{array}{l}\text { Comparar o máximo torque voluntário isométrico e o } \\
\text { recrutamento de unidades motoras do quadríceps } \\
\text { após estimulação elétrica em indivíduos pós-AVE. }\end{array}$ & $\begin{array}{l}\text { Melhora considerável do } \\
\text { controle motor. }\end{array}$ \\
\hline Salm et al. & 2006 & $\begin{array}{l}\text { Comparar o efeito de } 3 \text { métodos de estimulação } \\
\text { elétrica para reduzir a espasticidade do tríceps sural } \\
\text { em pacientes com lesão da medula espinhal completa } \\
\text { e investigar o efeito de transição. }\end{array}$ & $\begin{array}{l}\text { Estimulação do tríceps } \\
\text { sural e reduz o MAS } \\
\text { (escala de Ashworth } \\
\text { modificada) para esse } \\
\text { músculo específico. }\end{array}$ \\
\hline Carvalho; Cliquet & 2006 & $\begin{array}{l}\text { Avaliar o efeito do treinamento de marcha, com } \\
\text { estimulação elétrica neuromuscular (EENM), nos } \\
\text { sistemas esquelético e cardiorrespiratório de } \\
\text { tetraplégicos. }\end{array}$ & $\begin{array}{l}\text { Aumento da taxa de } \\
\text { formação óssea e da } \\
\text { capacidade aeróbia. }\end{array}$ \\
\hline Lima et al. & 2008 & $\begin{array}{l}\text { Verificar a força muscular e a resistência ao } \\
\text { movimento dos músculos flexores e extensores do } \\
\text { joelho de pacientes hemiplégicos após EENM e } \\
\text { exercícios isotônicos }\end{array}$ & $\begin{array}{l}\text { Diminuição } \\
\text { espasticidade e aumento } \\
\text { de força dos extensores } \\
\text { do joelho do lado } \\
\text { parético. }\end{array}$ \\
\hline Silva et al. & 2010 & $\begin{array}{l}\text { Analisar a fadiga e a força muscular do músculo } \\
\text { bíceps braquial, após a EENM com frequência de } 50 \text { e } \\
2000 \mathrm{~Hz} \text { (protocolo } 1 \text { e } 2 \text { respectivamente). }\end{array}$ & $\begin{array}{l}\text { Não houve evidência de } \\
\text { fadiga, porém constatou- } \\
\text { se melhora da força } \\
\text { muscular. }\end{array}$ \\
\hline
\end{tabular}


Tabela 2 - Distribuição dos artigos relacionados à EENM antagonista

\begin{tabular}{|c|c|c|c|}
\hline AUTOR & ANO & OBJETIVO & RESULTADOS \\
\hline Mañó et al. & 2011 & $\begin{array}{l}\text { Analisar o efeito da eletroestimulação } \\
\text { sobre a ADM e força da mão em } \\
\text { pacientes com hemiplegia espástica } \\
\text { após um AVC. }\end{array}$ & $\begin{array}{l}\text { Melhoras significativas }(p<0,05) \text { na ADM } \\
\text { e força. }\end{array}$ \\
\hline Sahin et al. & 2011 & $\begin{array}{l}\text { Avaliar a eficácia da EENM sobre a } \\
\text { espasticidade dos flexores do punho } \\
\text { após um evento cerebrovascular. }\end{array}$ & $\begin{array}{l}\text { EENM juntamente com alongamento dos } \\
\text { músculos de extensores de punho foi mais } \\
\text { eficaz na redução da espasticidade } \\
\text { quando comparado ao alongamento } \\
\text { sozinho. }\end{array}$ \\
\hline Lin et al. & 2011 & $\begin{array}{l}\text { Investigar a eficácia em longo prazo de } \\
\text { EENM no aperfeiçoamento da } \\
\text { recuperação motor de pacientes pós- } \\
\text { AVE. }\end{array}$ & $\begin{array}{l}\text { Melhora do controle motor persistente por } \\
6 \text { meses após tratamento. }\end{array}$ \\
\hline Knutson; Chae & 2010 & $\begin{array}{l}\text { Avaliar a viabilidade de melhorar a } \\
\text { dorsiflexão ativa do tornozelo com } \\
\text { EENM. }\end{array}$ & $\begin{array}{l}\text { Melhora do controle motor e ADM do } \\
\text { tornozelo. }\end{array}$ \\
\hline Hsu et al. & 2010 & $\begin{array}{l}\text { Investigar os efeitos de diferentes } \\
\text { doses de EENM em função da } \\
\text { extremidade superior em pacientes } \\
\text { pós-AVC. }\end{array}$ & Melhora da função motora. \\
\hline
\end{tabular}

\section{DISCUSSÃO}

A estimulação elétrica, comumente empregada na reabilitação neurológica, pode ser utilizada para estimular o Sistema Nervoso Central e Periférico, com a finalidade de reduzir a espasticidade. (SANTOS; NETO, 2006; SALM et al., 2006).

Outros resultados esperados incluem o aumento dos efeitos funcionais da toxina botulínica, quando utilizada com o objetivo de aumentar a força dos músculos paréticos, além de contrabalanceio da paralisia muscular, com consequente melhora da marcha (SANTOS; NETO, 2006; BHATKA, 2000; TEIVE, 1998); aumento da taxa de formação óssea e capacidade aeróbia (CARVALHO; CLIQUET, 2006); melhora do controle motor (NEWSAM; BACKER, 2004; CHAN, 2009).

Sua aplicação consiste na estimulação de um músculo com comprometimentos funcionais, a fim de obter-se uma contração útil. O mecanismo de ação dá-se pela despolarização do nervo correspondente ao ponto motor utilizado, produzindo uma resposta sincronizada das unidades motoras. Ocorre ainda, contantes aferências ao SNC ativando a musculatura local e igualmente os mecanismos reflexos necessários à reorganização da atividade motora, e, além disso, há uma redução do tônus muscular agonista, pelo mecanismo de inibição recíproca (TEIVE, 1998; LIANZA, 2003).

A eficácia da EENM no tratamento de indivíduos hemiplégicos espásticos tem sido comprovada em diversos estudos, utilizando-se diferentes protocolos de aplicação (JERONIMO, 2010; SILVA, 2010; BECK et al., 2010; SILVA et al., 2010; NUNES et al., 2008; LIMA et al. 2008; AKINBO et al., 2007).

A estimulação do músculo antagonista ao espástico, como descrito anteriormente, baseia-se no mecanismo de inibição recíproca, por meio das fibras la, enquanto a estimulação do agonista fundamenta-se no mecanismo de inibição autógena, ou seja, do próprio motoneurônio alfa do músculo 
estimulado (SILVA, 2011).

A inibição recíproca ocorre quando há estimulação do neurônio motor alfa do músculo agonista com consequente ativação de um interneurônio, em nível medular, porém com função inibitória exercida sobre o neurônio motor alfa do músculo antagonista. Assim, enquanto o músculo agonista é ativado, o músculo antagonista é inibido (COHEN, 2001).

O mecanismo de inibição recíproca tornar-se-ia falho, quando o músculo agonista sob ação eferente do motoneurônio alfa, apresentar seu tônus basal recuperado, interferindo no alongamento (COHEN, 2001).

Em contrapartida, a inibição autógena, além de inibir o motoneurônio alfa do músculo gerador de força, provê uma excitação do músculo antagonista, por meio de sinapses com interneurônio lb excitatório, sugerindo um alongamento mais eficaz. Fisiologicamente os órgãos tendinosos de Golgi (OTG), inervados por fibras sensoriais do tipo lb, detectam a força de contração muscular influenciando o grupo neuronal motor homônimo por meio de um interneurônio do tipo lb inibitório com ação sob o motoneurônio a (alfa) do músculo agonista. Simultaneamente, os neurônios motores antagonistas são facilitados (excitação recíproca) por meio de sinapses com interneurônio lb excitatório (COHEN, 2001).

De acordo com os resultados obtidos dos artigos pesquisados, a EENM mostrou-se eficaz tanto na aplicação sobre o músculo antagonista como sobre o agonista. Todavia, sob o ponto de vista fisiológico da contração muscular, sugerem-se melhores efeitos após a EENM agonista.

Silva (2011) realizou EENM no músculo bíceps braquial de 15 indivíduos, vítimas de AVE com quadro clínico de hemiparesia espástica, verificando uma redução da espasticidade com efeitos predominantemente tardios, além de redução da resistência à movimentação passiva do músculo antagonista ao espástico.

Paula et al. (2010) observaram redução da espasticidade, do tempo gasto e da velocidade da marcha em percurso prédeterminado, após aplicação de EENM no músculo reto femoral de dois indivíduos hemiparéticos, sugerindo ser esta uma alternativa eficaz no processo de reabilitação de indivíduos com hipertonia espástica do reto femoral, proporcionando melhora da funcionalidade e independência.

Carvalho e Cliquet (2006) avaliaram a marcha de 11 indivíduos, os quais realizaram EENM no músculo quadríceps. Os resultados demonstraram um aumento da taxa de formação óssea e da capacidade aeróbia.

\section{CONCLUSÃO}

Os artigos analisados sugerem que tanto a EENM antagonista quanto a agonista são um método de tratamento útil na reabilitação de indivíduos hemiplégicos espásticos, porém há escassez literária quanto a ensaios clínicos comparativos de tais métodos, sendo este um campo de estudo importante para a efetividade da intervenção fisioterapêutica, visando à reabilitação dos pacientes hemiplégicos. 


\section{REFERÊNCIAS BIBLIOGRÁFICAS}

AFIFI, A. K.; BERGMAN, R. A. Neuroanatomia Funcional: texto e atlas. São Paulo: Roca, 2007.

AKINBO, S. R. A. et al Comparision of the Effect of Neuromuscular Electrical Stimulation and Cryotherapy on Spasticity and Hand Function in Patients with Spastic Cerebral Palsy Nigerian Medical Practitioner. v. 51, n. 6, p. 128-132, 2007.

BHATKA, B. B. Management of Spasticity in Stroke. British Medical Bulletin. v. 56, n. 2, p. $476-485,2000$.

BECK, E. K. et al. Efeitos da estimulação elétrica funcional no controle neuromuscular artificial. Rev. Neurocienc. In press, 2010.

CARVALHO, D. C. L.; CLIQUET, A. J. Investigação das alterações osteometabólicas e cardio-respiratórias ocorridas após o treinamento de marcha sob estimulação elétrica neuromuscular em pacientes tetraplégicos. Acta ortop. bras., v.14, n. 3, 2006.

CHAN, M. K.; TONG, R. K.; CHUNG, K. Y. Bilateral Upper Limb Training With Functional Electric Stimulation in Patients With Chronic Stroke. Neurorehabilitation and Neural Repair. v. 23, n. 4, p.357-365, may.2009.

COHEN, H. Neurociência para fisioterapeutas. São Paulo: Manole, 2001.

FREITAS, S. T. T. Análise comparativa em dois diferentes métodos de avaliação da espasticidade em indivíduos com lesão da medula espinhal: Escala de Ashworth Modificada X Movimento Passivo Contínuo no equipamento isocinético. Dissertação (Mestrado em Ciências Biológicas) -
Instituto de Pesquisa e Desenvolvimento, Universidade do Vale do Paraíba, São José dos Campos, 2005.

GLANZ, M. et al. Functional electrostimulation in post-stroke rehabilitation: A meta-analysis of the randomized controlled trials. Archives of Physical Medicine and Rehabilitation, v. 77, jun.1996.

GLINSK, J.; HARVEY, L. Efficacy of electrical stimulation to increase muscle strength in people with neurological conditions: a systematic review.

Physiotherapy Research International Physiother. Res. Int. v. 12, n. 3, p. 175-194, jul.2007.

HSU, A. L.; TANG, P. F.; JAN, M. H. TestRetest Reliability of Isokinetic Muscle Strength of the Lower Extremities in Patients With Stroke. Arch. Phys Med Rehabil, v. 83, p. 1130-36, 2002.

HSU, S. S.; HU, M. H.; WUANG, Y. H.; YIP, P. K.; CHIU, J. W.; HSIEH, C. L. DoseResponse Relation Between Neuromuscular Electrical Stimulation and Upper-Extremity Function in Patients With Stroke. Stroke, v. 41, n. 4,p. 821-4, apr.2010.

JERÔNIMO, B. P. et. al. Spatio-Temporal Gait Variables Of Children With Cerebral Palsy Undergoing Eletrostimulation In The Anterior Tibial Muscle. Rev. bras. fisioter., v. 11, n. 4, 2007.

KNUTSON, J. S.; CHAE, J. A novel neuromuscular electrical stimulation treatment for recovery of ankle dorsiflexion in chronic hemiplegia: a case series pilot study. Am J Phys Med Rehabil., v. 89, n. 8, p. 672-82, aug.2010.

LEITÃO, A. V. et al. Espasticidade: Revista Univap, São José dos Campos-SP, v. 17, n. 30, dez.2011. ISSN 2237-1753 
Avaliação clínica. Projeto Diretrizes. Associação médica Brasileira e Conselho Federal de Medicina. São Paulo: Sociedade Brasileira de Medicina Física e Reabilitação, p. 8, 2006.

LIANZA, S. Medicina de Reabilitação. 3 ed. Editora Guanabara, 2001. p. 299.

Estimulação elétrica funcional: FES e reabilitação. 2. ed. São Paulo: Ateneu; 2003.

LIMA, M. O. et al. Efecto de la estimulación elétrica neuromuscular y de los ejercicios isotônicos em los músculos flexores y extensores de la rodilla em pacientes hemipléjicos. Revista de Neurología, v. 46, n. 3, p. 135-138, 2008.

LIMA, F. P. S. Efeito da Estimulação Elétrica Neuromuscular (EENM) e dos Exercícios Isotônicos do Fortalecimento dos Músculos Flexores e Extensores de Joelho em Pacientes Hemiplégicos. Dissertação (Mestrado em Ciências Biológicas) - Instituto de Pesquisa e Desenvolvimento, Universidade do Vale do Paraíba, 2005.

LIN, Z.; YAN, T. Long-term effectiveness of neuromuscular electrical stimulation for promoting motor recovery of the upper extremity after stroke. J Rehabil. Med., 43: p. 506-510, 2011.

MACHADO, A. B. M. Neuroanatomia Funcional. 2 ed. Belo Horizonte: Ateneu, 1993. p. 87-92.

MAÑÓ, S. T., SALOM, T.; TOMÁS, J. M.; MELÉNDEZ, M. J. C.; FUENTE, F. T. L. Electrical stimulation in the treatment of the spastic hemiplegic hand after stroke: a randomized study. Med Clin (Barc), v. 137, n. 7, p. 297-301, sep.2011.

MINUTOLI, V. P. et al. Efeito do movimento passivo contínuo isocinético na hemiplegia espástica. Acta Fisiátrica, v. 14, n. 3, p. 142-148, 2007.

MIYAZAKI, E. G. et al. Influencia da estimulação elétrica funcional para adequação de tono muscular e controle motor em hemiplégicos. INTELLECTUS Revista Acadêmica Digital do Grupo POLIS Educacional, ano 4, n. 5, jul./dez., 2008

NEWSAM, C. J.; BAKER, L. L. Effect of an Electric Stimulation Facilitation Program on Quadriceps Motor Unit Recruitment After Stroke. Arch Phys Med Rehabil, v. 85, p. 2040-2045, dec./2004.

NORONHA, M. A. O efeito da Estimulação Elétrica Neuromuscular (EENM) no músculo tibial anterior do rato. Revista Bras Fisiot., v. 2, n. 2, 1997.

NUNES, L. C. B. G.; QUEVEDO, A. A. F.; MAGDALON, E. C. Effects of neuromuscular electrical stimulation on tibialis anterior muscle of spastic hemiparetic children. Rev

Bras Fisioter, v. 12, n. 4, 2008.

NUYENS, G. E. et al. Reduction of spastic hypertonia during repeated passive knee movements in stroke patients. Arch Phys Med Rehabil, v. 83, p. 930-935, 2002.

O'SULLIVAN. Fisioterapia Avaliação e Tratamento. 4. ed. São Paulo: Manole, 2004.

PAULA, A. A. et al. Reabilitação motora em hemiplégicos espásticos após tratamento com estimulação elétrica neuromuscular. Revista UniVap, v. 16, n. 28, p. 112-118, 2010. 
PUPULIN, E.; ZECCHINI, E. Promovendo qualidade de vida após acidente vascular cerebral: um guia para fisioterapeutas e profissionais de atenção primária à saúde/ Organização Mundial da Saúde - Porto Alegre: Artmed, 2003.

SAHIN, N.; UGURLU, H.; ALBAYRAK, I. The efficacy of electrical stimulation in reducing the post-stroke spasticity: a randomized controlled study. Disabil Rehabil. 2012. v. 34, n. 2, p. 151-6. Oct./2011.

SANTOS, L.; NETO, T. Efetividade da Fisioterapia na Restituição da Funcionalidade dos Indivíduos com Espasticidade pós-AVC. Monografia (Licenciatura bi-etápica em Fisioterapia) Escola Superior de Saúde Atlântica, 2005/2006.

SALM, A. V. et al. Comparison of electric stimulation methods for reduction of tríceps surae spasticity in spinal cord injury. Arch Phys Med Rehabil, v. 87, p. 222-8, 2006.

SCHUSTER, R. C.; SANT, C. R.; DALBOSCO, V. Efeitos da estimulação elétrica funcional (FES) sobre o padrão de marcha de um paciente hemiparético. Acta Fisiatr., n. 2, v. 14, 2007.

SILVA, D. D. Análise do Torque e RMS do Membro Superior Parético Espástico Submetido à Crioterapia e Estimulação Elétrica Neuromuscular. Dissertação (mestrado em Engenharia Biomédica) Instituto de Pesquisa e Desenvolvimento, Universidade do Vale do Paraíba, 2011.

SILVA, S. M. et al. Estudo da fadiga muscular pela eletromiografia e força muscular, após dois protocolos de estimulação elétrica funcional.

ConScientiae Saúde, n. 2, v. 9, 2010.

SOMMERFELD, D. K. et al. Spasticity After Stroke: Its Occurrence and Association With Motor Impairments and Activity Limitations.

Stroke, 35, p. 134-139, jan./2004.

SORINOLA, I. O.et al. Electromyographic Response to Manual Passive Stretch of the Hemiplegic Wrist: Accuracy, Reliability, and Correlation With Clinical Spasticity Assessment and Function. Neurorehabil Neural Repair. v. 23, december, 2009.

TEIVE, A. G. H.; ZONTA, M.; KUMAGAI, Y. Tratamento da espasticidade - uma atualização. Arq Neuropsiquiatria, n. 3, v. 2, p. 852-858, 1998.

UMPHRED, D. A. Fisioterapia Neurológica. 2. ed. São Paulo: Manole, 1994, p. 195-196.

WANG, R. Y. et al. Effects of surface Spinal Cord Stimulation on Spasticity and Quantitative Assessment of muscle tone in Hemiplegic Patients. Am J Phys. Med Rehabil., v. 77, n. 4. jul./aug., 1998. 